Nota técnica

Volumen 31(3):729-741. Septiembre-diciembre, 2020 e-ISSN 2215-3608, doi:10.15517/am.v31i3.40302 http://www.revistas.ucr.ac.cr/index.php/agromeso

\title{
Caracterización fenotípica de tres cultivares de ají dulce (Capsicum chinense Jacq.) venezolano ${ }^{1}$
}

\section{Phenotypic characterization of three Venezuelan sweet pepper (Capsicum chinense Jacq.)}

\author{
Maryleidi Quevedo ${ }^{2}$,Hernán Laurentin ${ }^{2}$
}

1 Recepción: 13 de enero, 2020. Aceptación: 25 de mayo, 2020. Este trabajo formó parte del trabajo de grado de la primera autora para optar al título de Ingeniera Agrónoma en la Universidad Centroccidental Lisandro Alvarado. Fue un trabajo autofinanciado por ambos autores.

2 Universidad Centroccidental Lisandro Alvarado, Facultad de Agronomía, Departamento de Ciencias Biológicas, Barquisimeto, Venezuela. maryleidi009@hotmail.com (https://orcid.org/0000-0002-1120-7002), hlaurentin@ucla.edu.ve (https://orcid.org/0000-0001-6222-3566).

\section{Resumen}

Introducción. Las especies cultivadas del género Capsicum, entre ellas C . chinense, son conocidas por la pungencia de sus frutos, sin embargo, existen muchos tipos de este ají que no presentan pungencia. La cocina venezolana es definida por el sabor característico de una clase de estos ajíes que no presentan pungencia, los cuales son llamados ajíes dulces, entre estos, los más conocidos y usados son los cultivares Rosita, Pepón y Llanero. Objetivo. Caracterizar los cultivares de ají Rosita, Pepón y Llanero mediante 53 caracteres morfológicos y determinar la relación fenética existente entre ellos. Materiales y métodos. En un diseño de bloques al azar con tres tratamientos (cultivares) y cinco repeticiones, se dispusieron 45 plántulas de cada cultivar en La Vigía, municipio Jiménez del estado Lara, Venezuela, en agosto del año 2017. El trasplante se efectuó en hileras separadas a 0,50 m, y las plántulas se distanciaron $0,50 \mathrm{~m}$. sobre la hilera. Sobre las tres plantas de la hilera central de cada unidad experimental (quince plantas en total para cada cultivar) se determinó la categoría o el valor de atributos de tallo, hoja, flor, fruto y semilla. Resultados. El $42 \%$ de estos atributos resultaron variables entre las plantas evaluadas, quince de ellos fueron categóricos y siete cuantitativos, y se detectaron en estos diferencias significativas $(p<0,05)$ entre cultivares. Características de flor y semilla no presentaron variación entre cultivares, mientras que tallo y fruto presentaron las mayores variaciones. En conjunto, las características morfológicas que presentaron variación permitieron identificar a cada uno de los cultivares y por tanto, discriminarlos. Conclusión. La variación identificada en las características del fruto es indicativa del potencial que se tiene para mejorar genéticamente el ají dulce venezolano en su parte comercial.

Palabras clave: agrobiodiversidad, descriptor morfológico, identidad genética, recurso fitogenético.

\begin{abstract}
Introduction. The cultivated species of the genus Capsicum, among them $C$. chinense, are known for the pungency of their fruits, however, there are several types of this pepper that do not present pungency. Venezuelan cuisine is defined by the characteristic flavor of a class of peppers that do not present pugency, which are called
\end{abstract}


sweet peppers. Among these, the best known and most used are the cultivars Rosita, Pepon, and Llanero. Objective. To characterize the Rosita, Pepon, and Llanero chilli pepper cultivar by means of 53 morphological characteristics and to determine the phenetic relationship among them. Material and methods. In a randomized block design with three treatments (cultivars) and five repetitions, 45 seedlings of each cultivar were arranged in La Vigia, Jimenez municipality of Lara state, Venezuela, in August 2017. The transplant was carried out in separate rows at $0.50 \mathrm{~m}$, and the seedlings were spaced $0.50 \mathrm{~m}$ apart on the row. On the three plants of the central row of each experimental unit (fifteen plants in total for each cultivar) the category or value of attributes of stem, leaf, flower, fruit, and seed were determined. Results. Forty-two percent of these attributes were variable among the plants evaluated, fifteen of them were categorical and seven quantitative, and significant differences $(\mathrm{p}<0.05)$ were detected among cultivars. Flower and seed characteristics showed no variation among cultivars, while stem and fruits showed the greatest variation. As a whole, the morphological characteristics that presented variation allowed to identify each one of the cultivars and therefore, to discriminate them. Conclusion. The variation identified in the characteristics of the fruit is indicative of the potential for genetic improvement of the Venezuelan sweet pepper in its commercial part.

Keywords: agrobiodiversity, genetic identity, morphological descriptor, plant genetic resources.

\section{Introducción}

Los recursos fitogenéticos son las plantas o partes de ellas con potencial regenerativo, que tienen valor económico, social o científico para la humanidad (Laurentin, 2009), y representan los componentes más importantes de la agrobiodiversidad (Upadhyaya et al., 2008). Ante tal importancia, la humanidad se ha abocado desde hace décadas a su conservación, ya sea in situ o ex situ. La conservación de estos recursos genéticos permite contar con material vegetal suficiente para estudios de diversidad y evolución de especies vegetales (Holmes et al., 2016), la conservación per se (Hallewood et al., 2018), acceder fácilmente a introducciones de una especie para mejorar genéticamente sus poblaciones y obtener nuevos cultivares (Govindaraj et al., 2015), además de tener un conocimiento integral de recursos vegetales tradicionales, bien sea para alimentación o como medicina (Eldeen et al., 2016).

Uno de los objetivos de una caracterización morfológica es identificar inequívocamente un cultivar, de esta manera se podrá tener parte del conocimiento integral de los recursos fitogenéticos tradicionales (Eldeen et al., 2016). Es así como la caracterización morfológica de varios cultivares también buscará la diferenciación entre ellos. Otro de los objetivos al caracterizar materiales vegetales es conocer la diversidad genética existente para cada carácter y así, conocer el potencial existente para hacer mejoramiento genético (Laurentin, 2009; Govindaraj et al., 2015).

Entre los primeros esfuerzos por caracterizar los recursos fitogenéticos, se encuentra la evaluación de características morfológicas. Por la desventaja que presentaba la plasticidad fenotípica de estos atributos así como la ocurrencia de pocos caracteres que permitieran diferenciar fenotipos dentro de una especie, se inició la caracterización molecular mediante marcadores de ADN, los cuales tienen el potencial de diferenciación entre plantas de una misma especie, además de ser absolutamente insensibles al ambiente (Laurentin, 2009).

A pesar de la ventaja de la caracterización molecular sobre la morfológica, Govindaraj et al. (2015) consideraron que los marcadores morfológicos son de uso obligatorio en el campo para poder distinguir cultivos de plantas fuera de tipo o de plantas espontáneas; estos autores consideraron que esta caracterización se hace con atributos que pueden ser fácilmente observables tales como el color de la flor, la forma de semilla, el hábito de crecimiento y la pigmentación. 
Entre las instituciones que han logrado esfuerzos importantes en la sistematización de la caracterización morfológica está el International Plant Genetic Resources Institute (IPGRI), quienes lograron elaborar descriptores morfológicos para muchas especies vegetales, y en colaboración con Asian Vegetable Research and Development Center, y Centro Agronómico Tropical de Investigación y Enseñanza, publicaron descriptores morfológicos para Capsicum sp. (IPGRI et al., 1995). Los atributos morfológicos usados en una caracterización deben permitir una rápida y fácil discriminación entre genotipos, deben ser altamente heredables y fácilmente identificables.

$\mathrm{El}$ género Capsicum posee cinco especies domesticadas, las cuales son C. annuum, C. frutescens, C. chinense, C. baccatum y C. pubescens (Shirasawa et al., 2019). En estas especies se han hecho caracterizaciones morfológicas basadas en el descriptor de IPGRI et al. (1995) (Sudré et al., 2010; Baba et al., 2016; Carvalho et al., 2017; dela-Cruz-Lázaro et al., 2017; Cardoso et al., 2018; Olatunji y Afolayan, 2019). Los frutos que se derivan de estas especies son comúnmente llamados chiles, pimientos o ajíes.

El ají, específicamente el perteneciente a la especie Capsicum chinense, es mundialmente famoso por la pungencia de sus frutos, especialmente la del chile habanero. C. chinense es una especie originaria de la cuenca del río Amazonas (Eshbaugh, 1980), incluye tipos no pungentes y presenta una gran diversidad genética en América del Sur con una gran variabilidad genética en Venezuela, país considerado un centro de diversidad (Moses y Umaharan, 2012). Uno de los tipos de C. chinense presente en Venezuela es el ají dulce, el cual es un ingrediente característico de la cocina venezolana en la elaboración de guisos, como condimento para carnes y pescados y como ingrediente imprescindible en platos tradicionales (Colivet et al., 2006; Pastrán, 2019). Es denominado ají dulce por no poseer pungencia.

Como ají dulce en la cocina venezolana se encuentran tres tipos que son totalmente diferenciables por la forma y color de su frutos, situación que es común en la especie, ya que Capsicum chinense ha sido reportada con una amplia diversidad genética, especialmente en lo relacionado a su amplia variabilidad en formas, colores y pungencia del fruto (Baba et al., 2016). Los materiales venezolanos a que se hace referencia han sido tradicionalmente nombrados como ají Rosita, ají Pepón y ají Llanero, de los cuales no se tiene reporte de obtención. La producción de plántulas de estos materiales es llevada a cabo por productores independientes, y son comercializadas bajo los nombres indicados. A pesar de la importancia etnobotánica de estos ajíes, especialmente de su importancia culinaria en Venezuela, poco se conoce acerca de estos ajíes más allá del conocimiento tradicional. Los esfuerzos que se han hecho para el conocimiento del ají dulce venezolano han sido mas que todo con respecto a labores hortícolas tales como tiempo óptimo de trasplante (Montaño, 2000a), métodos para producción de plántulas (Montaño, 2000b), efecto de estrés hídrico sobre la producción (Jaimez, 2000), poda en plantas (Jaimez et al., 2002), control de patógenos (Cedeño et al., 2003), necesidades de riego (Gil-Marín et al., 2012), y tratamiento pre germinativo (Andrade y Laurentin, 2015) pero no existen reportes sobre la identidad genética de estos cultivares.

El objetivo de este trabajo fue caracterizar los tres cultivares de ají Rosita, Pepón y Llanero mediante 53 caracteres morfológicos y determinar la relación fenética existente entre ellos.

\section{Materiales y métodos}

\section{Material vegetal y localidad}

Se obtuvieron 45 plántulas de ají dulce (Capsicum chinense Jacq.) en agosto del año 2017, de tres variedades conocidas como Rosita, Pepón y Llanero; estas plántulas se adquirieron en un vivero comercial de Quíbor, municipio Jiménez, estado Lara. Las plántulas se trasplantaron en una parcela de la localidad La Vigía, en el kilómetro 9 de la vía Quíbor, El Tocuyo, municipio Jiménez, estado Lara, a $705 \mathrm{msnm}$ y de coordenadas $09^{\circ} 53^{`} \mathrm{~N}$ y $69^{\circ} 39^{\prime} \mathrm{W}$. 


\section{Diseño experimental}

En agosto de 2017 las plántulas se trasplantaron siguiendo un diseño de experimentos de bloques al azar con cinco repeticiones y tres tratamientos representados por los materiales Rosita, Pepón y Llanero. Cada parcela experimental consistió en tres camellones distanciados 0,50 m entre sí, en cada uno de ellos se trasplantaron tres plántulas separadas $0,50 \mathrm{~m}$ entre sí, por tanto, cada parcela experimental estuvo constituida por nueve plantas. Las plantas se regaron mediante un sistema por goteo, se aplicó una única fertilización con urea al momento del aporque, la tercera semana después del trasplante, a razón de $80 \mathrm{~kg} \mathrm{ha}^{-1}$.

\section{Recolección de datos}

Entre agosto y noviembre de 2017 se evaluaron 53 caracteres morfológicos incluidos en el descriptor de Capsicum spp. (IPGRI et al., 1995). Estas variables se evaluaron en las tres plantas ubicadas en el surco central de cada unidad experimental, en el momento y la forma en que lo sugiere IPGRI et al. (1995) para cada carácter. Los atributos morfológicos evaluados fueron diecinueve vegetativos (seis de tallo, siete de hoja, y seis de la planta en su conjunto), trece de flores y floración, diecisiete de frutos y fructificación, y cuatro de semilla. Las variables evaluadas son mostradas en los Cuadros 1 y 2.

\section{Análisis estadístico}

Se realizaron dos tipos de análisis, uno univariado y el otro multivariado. El análisis univariado difirió entre las variables categóricas y las variables cuantitativas. En las variables categóricas se consideraron los datos para cada una de las quince plantas evaluadas de cada cultivar, y se realizó una distribución de frecuencias así como la determinación de la mediana. Para las variables cuantitativas, el valor de cada unidad experimental estuvo representado por el promedio de cada variable sobre las tres plantas evaluadas; con estos valores se realizaron análisis de varianza. En aquellas variables donde hubo diferencias estadísticas $(\mathrm{p}<0,05)$, se realizó una prueba de medias de Tukey entre cultivares. Todos estos análisis se llevaron a cabo mediante el programa Statistix 8 .

Para el análisis multivariado, cada planta evaluada representó una unidad taxonómica operacional (OTU). Para esto, en la matriz de datos se estandarizaron sus variables, restándole a cada valor el promedio de estas y dividiéndolas entre su desviación estándar. Posteriormente, se determinó la correlación entre las variables. Con la matriz que se obtuvo se calcularon los autovectores, luego se proyectaron los valores obtenidos estandarizados sobre el espacio multidimensional. El ordenamiento de cada una de las parcelas experimentales permitió establecer cualitativamente la magnitud de la diversidad intravarietal con respecto a la intervarietal, lo cual se hace necesario para constatar la identidad genética de cada cultivar evaluado.

\section{Resultados}

De los 53 caracteres morfológicos evaluados, quince categóricos resultaron con variación entre las 45 plantas evaluadas, y en siete cuantitativos hubo diferencias estadísticas significativas $(\mathrm{p}<0,05)$ entre cultivares. En seis de las variables categóricas evaluadas pertenecientes a los rasgos morfológicos vegetativos, no existió variabilidad entre las quince plantas evaluadas de cada uno de los cultivares (Cuadro 3), lo cual sí ocurrió en ocho de las variables (cuatro de tallo, uno de hoja y tres de planta en conjunto) (Cuadro 4). En el $60 \%$ de las variables vegetativas cuantitativas no se detectaron diferencias estadísticas entre cultivares (Cuadro 5). Los doce atributos categóricos medidos en flor y floración resultaron invariables entre las 45 plantas evaluadas (Cuadro 3); la variable 
Cuadro 1. Variables de planta, tallo, hoja y flor medidas para caracterizar tres variedades: Rosita, Pepón y Llanero de ají dulce (Capsicum chinense Jacq.) venezolano, en La Vigía, municipio Jiménez, estado Lara, Venezuela. 2017.

Table 1. Variables of plant, stem, leaf, and flower measured to characterize three varieties: Rosita, Pepón, and Llanero of Venezuelan sweet peppers (Capsicum chinense Jacq.) in La Vigía, Jimenez municipality, Lara state, Venezuela. 2017.

\begin{tabular}{|c|c|}
\hline Variable & Categorías y codificación \\
\hline 2.1. Ciclo de vida & 1. Anual. 2. Bianual. 3. Perenne \\
\hline 2.2. Color del tallo & 1. Verde. 2. Verde con rayas púrpura. 3. Morado. 4. Otro \\
\hline 2.3. Antocianina del nudo & 1. Verde. 3. Morado. 5. Morado claro. 7. Morado oscuro. \\
\hline 2.4. Forma del tallo & 1. Cilíndrico. 2. Angular. 3. Achatado. \\
\hline 2.5. Pubescencia del tallo & 3. Escasa. 5. Intermedia. 7. Densa \\
\hline 2.6. Altura de la planta $(\mathrm{cm})$ & 1. $<25.2 .26-45.3 .46-65.4 .66-85.5 .>85$ \\
\hline 2.7. Hábito de crecimiento de la planta & 3. Postrada. 5. Intermedia. 7. Erecta. 9. Otro \\
\hline \multicolumn{2}{|l|}{ 2.8. Ancho de la planta (cm) } \\
\hline \multicolumn{2}{|l|}{ 2.9. Longitud del tallo $(\mathrm{cm})$} \\
\hline \multicolumn{2}{|l|}{ 2.10. Diámetro del tallo $(\mathrm{cm})$} \\
\hline 2.11. Densidad de ramificación & 3. Escasa. 5. Intermedia. 7. Densa \\
\hline 2.12. Macollamiento & 3. Escaso. 5. Intermedio. 7. Denso. \\
\hline 2.13. Densidad de hojas & 3. Escasa. 5. Intermedio. 7. Denso \\
\hline 2.14. Color de hoja & $\begin{array}{l}\text { 1. Amarillo. 2. Verde claro. 3. Verde. 4. Verde oscuro. 5. Morado claro. 6. Morado. } 7 . \\
\text { Jaspeado. 8. Otro }\end{array}$ \\
\hline 2.15. Forma de la hoja & 1. Deltoide. 2. Oval. 3. Lanceolada. \\
\hline 2.16. Margen de la lámina foliar & 1. Entera. 2. Ondulada. 3. Ciliada \\
\hline 2.17. Pubescencia de la hoja & 3. Escasa. 5. Intermedia. 7. Densa. \\
\hline \multicolumn{2}{|l|}{ 2.18. Longitud de la hoja madura $(\mathrm{cm})$} \\
\hline \multicolumn{2}{|l|}{ 2.19. Ancho de la hoja madura $(\mathrm{cm})$} \\
\hline \multicolumn{2}{|l|}{ 3.1. Días a la floración } \\
\hline 3.2. Número de flores por axila & 1. 1.2. 2.3.3. 4. Muchas en racimo. 5. Otro \\
\hline 3.3. Posición de la flor & 3. Pendiente. 5. Intermedia. 7. Erecta \\
\hline 3.4. Color de la flor & $\begin{array}{l}\text { 1. Blanco. 2. Amarillo claro. 3. Amarillo-verdoso. } 4 \text {. Morado con base blanca. } 5 \text {. } \\
\text { Morado con base blanca. } 6 \text {. Blanco con base púrpura. } 7 \text {. Blanco con margen púrpura. } 8 \text {. } \\
\text { Morado. } 9 \text {. Otro }\end{array}$ \\
\hline 3.5. Forma de la corola & 1. Redonda. 2. Acampanulada. 3. Otra \\
\hline 3.6. Longitud de la corola $(\mathrm{cm})$ & 1. $<1,5 \cdot 2 \cdot 1,5-2,5 \cdot 3 .>2,5$ \\
\hline 3.7. Color de las anteras & 1. Blanco. 2. Amarillo. 3. Azul pálido. 4. Azul. 5. Morado. 6. Otro. \\
\hline 3.8. Color del filamento & 1. Blanco. 2. Amarillo. 3. Verde. 4. Azul. 5. Morado claro. 6. Morado. 7. Otro. \\
\hline 3.9. Exserción del filamento & 3. Inserto. 5. Al mismo nivel. 7. Exserto. \\
\hline 3.10. Esterilidad masculina & 0. No. 1. Sí. \\
\hline 3.11. Pigmentación del cáliz & 0. Ausente. 1. Presente. \\
\hline 3.12. Margen del cáliz & 1. Entero. 2. Intermedio 3. Dentado. 4. Otro. \\
\hline 3.13. Constricción anular del cáliz & 0. Ausente. 1. Presente \\
\hline
\end{tabular}


Cuadro 2. Variables de fruto y semilla medidas para caracterizar tres variedades: Rosita, Pepón y Llanero, de ají dulce (Capsicum chinense Jacq.) venezolano, en La Vigía, municipio Jiménez, estado Lara, Venezuela. 2017.

Table 2. Fruit and seed variables measured to characterize three varieties: Rosita, Peón, and Llanero, of Venezuelan sweet pepper (Capsicum chinense Jacq.) in La Vigía, Jimenez municipality, Lara state, Venezuela. 2017.

\begin{tabular}{|c|c|}
\hline Variable & Categorías y codificación \\
\hline \multicolumn{2}{|l|}{ 4.1. Días a la fructificación } \\
\hline 4.2. Manchas antocianínicas & 0. Ausente. 1. Presente. \\
\hline 4.3. Color del fruto en estado intermedio & $\begin{array}{l}\text { 1. Blanco. 2. Amarillo. 3. Verde. 4. Anaranjado. 5. Morado. } 6 . \\
\text { Morado oscuro. } 7 \text {. Verde oscuro con morado oscuro. 8. Verde } \\
\text { claro con morado. 9. Otro. }\end{array}$ \\
\hline 4.4. Cuajado del fruto & 3. Bajo. 5. Intermedio. 7. Alto. \\
\hline 4.5. Color del fruto maduro & $\begin{array}{l}\text { 1. Blanco. 2. Amarillo-limón. 3. Amarillo-naranja pálido. } 4 . \\
\text { Amarillo-naranja. 5. Naranja pálido. 6. Naranja. } 7 \text {. Rojo claro. } \\
\text { 8. Rojo. 9. Rojo oscuro. 10. Morado. 11. Marrón. 12. Negro. } 13 . \\
\text { Otro. }\end{array}$ \\
\hline 4.6. Forma del fruto & $\begin{array}{l}\text { 1. Elongado. 2. Casi redondo. 3. Triangular. 4. Acampanulado. } 5 \text {. } \\
\text { Acampanulado y en bloque. } 6 \text {. Otro. }\end{array}$ \\
\hline \multicolumn{2}{|l|}{ 4.7. Longitud del fruto $(\mathrm{cm})$} \\
\hline \multicolumn{2}{|l|}{ 4.8. Ancho del fruto $(\mathrm{cm})$} \\
\hline \multicolumn{2}{|l|}{ 4.9. Peso del fruto $(\mathrm{g})$} \\
\hline \multicolumn{2}{|l|}{ 4.10. Longitud del pedicelo del fruto $(\mathrm{cm})$} \\
\hline \multicolumn{2}{|l|}{ 4.11. Espesor de pared del fruto $(\mathrm{mm})$} \\
\hline 4.12. Forma del ápice del fruto en unión con el pedicelo & 1. Agudo. 2. Obtuso. 3. Truncado. 4. Cordado. 5. Lobulado. \\
\hline 4.13. Cuello en la base del fruto & 0. Ausente. 1. Presente. \\
\hline 4.14. Forma del ápice del fruto & $\begin{array}{l}\text { 1. Puntiagudo. 2. Romo. 3. Hundido. 4. Hundido y puntiagudo. } \\
\text { 5. Otro. }\end{array}$ \\
\hline 4.15. Apéndice en el fruto vestigio de floración & 1. Ausente. 2. Presente. \\
\hline 4.16. Arrugamiento transversal del fruto & 1. Levemente corrugado. 2. Intermedio. 3. Muy corrugado. \\
\hline 4.17. Tipo de epidermis del fruto & 1 Lisa. 2 Semirrugosa. 3 Rugosa \\
\hline 5.1. Color de la semilla & 1. Amarillo oscuro. 2. Marrón. 3. Negro. 4. Otro \\
\hline 5.2. Superficie de la semilla & 1. Lisa. 2. Aspera. 3. Rugosa. \\
\hline 5.3. Tamaño de la semilla & 1. Pequeña. 2. Mediana. 3. Grande. \\
\hline 5.4. Número de semillas por fruto & $1<20.220-50.3>50$. \\
\hline
\end{tabular}

cuantitativa días a la floración presentó diferencias significativas $(\mathrm{p}<0,05)$ entre cultivares (Cuadro 5). Siete de las once características de fruto y fructificación designadas como categóricas presentaron variación entre las 45 plantas evaluadas (Cuadro 3). El $67 \%$ de los atributos morfológicos cuantitativos medidos sobre frutos y fructificación presentaron diferencias significativas $(\mathrm{p}<0,05)$ entre cultivares (Cuadro 5). Los atributos medidos sobre semilla no presentaron variación entre cultivares (Cuadro 3). 
Cuadro 3. Atributos morfológicos categóricos que resultaron invariables al evaluar cinco plantas de de las variedades Rosita, Pepón y Llanero de ají dulce (Capsicum chinense Jacq.) venezolano, en La Vigía, municipio Jiménez, estado Lara, Venezuela. 2017.

Table 3. Categorical morphological attributes that resulted without variation in the evaluation of five plants of the Venezuelan sweet pepper (Capsicum chinense Jacq.) varieties: Rosita, Pepon, and Llanero in La Vigía, Jiménez municipality, Lara state, Venezuela. 2017.

\begin{tabular}{|c|c|}
\hline Variable & Resultado \\
\hline 2.1. Ciclo de vida & Perenne \\
\hline 2.12. Macollamiento & Escaso \\
\hline 2.13. Densidad de la hoja & Denso \\
\hline 2.14. Color de la hoja & Verde \\
\hline 2.15. Forma de la hoja & Oval \\
\hline 2.16. Margen de la lámina foliar & Entera \\
\hline 3.2. Número de flores por axila & $\operatorname{Dos}(2)$ \\
\hline 3.3. Posición de la flor & Intermedia \\
\hline 3.4. Color de la flor & Amarillo claro \\
\hline 3.5. Forma de la corola & Redonda \\
\hline 3.6. Longitud de la corola $(\mathrm{cm})$ & Entre 1,5 y 2,5 \\
\hline 3.7. Color de las anteras & Blanco \\
\hline 3.8. Color del filamento & Verde \\
\hline 3.9. Exserción del estigma & Inserto \\
\hline 3.10. Esterilidad masculina & No \\
\hline 3.11. Pigmentación del cáliz & Presente \\
\hline 3.12. Margen del cáliz & Dentado \\
\hline 3.13. Constricción anular del cáliz & Ausente \\
\hline 4.13. Cuello en la base del fruto & Ausente \\
\hline 4.15. Apéndice en el fruto vestigio de floración & Ausente \\
\hline 4.16. Arrugamiento transversal del fruto & Levemente corrugado \\
\hline 4.17. Tipo de epidermis del fruto & Lisa \\
\hline 5.1. Color de la semilla & Amarillo oscuro \\
\hline 5.2. Superficie de la semilla & Rugosa \\
\hline 5.3. Tamaño de la semilla & Pequeña \\
\hline 5.4. Número de semillas por fruto & Más de 50 \\
\hline
\end{tabular}

Las variables que presentaron mayor variación fueron ancho de la planta y longitud del tallo para los cultivares Rosita y Llanero (Cuadro 5). El cultivar Pepón fue el que presentó en promedio menor variación para las características cuantitativas evaluadas, resultando ser el de menor variación en ocho de las doce variables registradas.

El gráfico bidimensional logrado por el análisis de componentes principales abarcó el $49 \%$ de la variación en los dos primeros ejes, $39 \%$ correspondió al primer eje y $10 \%$ correspondió al segundo. La variación intracultivar fue mucho más pequeña que la variación intercultivar, lo cual se ve reflejado en la Figura 1, donde se agruparon 
Cuadro 4. Mediana y distribución de frecuencias para los atributos morfológicos cualitativos no ordinales que presentaron variabilidad al ser medidas sobre quince plantas de las variedades Rosita, Pepón y Llanero de ají dulce (Capsicum chinense Jacq.) venezolano, en La Vigía, municipio Jiménez, estado Lara, Venezuela. 2017.

Table 4. Median and frequency distribution for morphological traits of the non-ordinal qualitative type, which presented variability when measured on fifteen plants of the Venezuelan sweet pepper (Capsicum chinense Jacq.) varieties: Rosita, Pepon, and Llanero. La Vigía, Jiménez municipality, Lara state, Venezuela. 2017.

\begin{tabular}{|c|c|c|c|c|c|c|}
\hline \multirow[t]{2}{*}{ Variable } & \multicolumn{3}{|c|}{ Mediana } & \multicolumn{3}{|c|}{$\begin{array}{l}\text { Distribución de frecuencias: primer número } \\
\text { indica la categoría, segundo número la frecuencia }\end{array}$} \\
\hline & Rosita & Pepón & Llanero & Rosita & Pepón & Llanero \\
\hline 2.2. Color del tallo & 1 & 4 & 4 & $1: 15$ & 4: 15 & 4: 15 \\
\hline 2.3. Antocianinas en tallo & 3 & 7 & 7 & $\begin{array}{l}3: 12 \\
7: 3\end{array}$ & 7: 15 & $7: 15$ \\
\hline 2.4. Forma del tallo & 1 & 2 & 2 & $\begin{array}{l}1: 12 \\
2: 3\end{array}$ & 2: 15 & 2: 15 \\
\hline 2.5. Pubescencia del tallo & 3 & 5 & 5 & $\begin{array}{l}3: 13 \\
5: 2\end{array}$ & 5: 15 & 5: 15 \\
\hline 2.6. Altura de la planta & 2 & 2 & 2 & $\begin{array}{l}1: 3 \\
2: 9 \\
3: 3\end{array}$ & 2: 15 & 2:15 \\
\hline 2.7. Hábito de crecimiento & 7 & 5 & 5 & $\begin{array}{l}5: 3 \\
7: 12\end{array}$ & 5: 15 & 5: 15 \\
\hline 2.11. Densidad de ramificación & 5 & 7 & 7 & $5: 15$ & $7: 15$ & $7: 15$ \\
\hline 2.17. Pubescencia de la hoja & 3 & 5 & 5 & $\begin{array}{l}3: 13 \\
5: 2\end{array}$ & 5: 15 & $5: 15$ \\
\hline 4.2. Manchas antocianínicas & 0 & 1 & 1 & $0: 15$ & 1: 15 & $1: 15$ \\
\hline $\begin{array}{l}\text { 4.3. Color del fruto en estado } \\
\text { intermedio }\end{array}$ & 3 & 8 & 7 & 3: 15 & $8: 15$ & $7: 15$ \\
\hline 4.4. Cuajado del fruto & 3 & 2 & 2 & $\begin{array}{l}2: 3 \\
3: 12\end{array}$ & $\begin{array}{l}2: 13 \\
3: 2\end{array}$ & $\begin{array}{l}\text { 2: } 9 \\
\text { 3: } 6\end{array}$ \\
\hline 4.5. Color del fruto maduro & 2 & 7 & 9 & 2: 15 & 7: 15 & 9: 15 \\
\hline 4.6. Forma del fruto & 4 & 5 & 1 & 4: 15 & 5: 15 & $1: 15$ \\
\hline $\begin{array}{l}\text { 4.12. Forma del ápice de fruto en } \\
\text { unión con el pedicelo }\end{array}$ & 4 & 5 & 4 & 4: 15 & $\begin{array}{l}4: 4 \\
5: 11\end{array}$ & 4: 15 \\
\hline 4.14. Forma del ápice del fruto & 2 & 3 & 1 & $2: 15$ & 3: 15 & $1: 15$ \\
\hline
\end{tabular}

las quince plantas evaluadas de cada cultivar, y se formaron tres grupos que correspondieron a cada uno de los tres cultivares evaluados. En el primer eje las variables más discriminantes entre cultivares fueron mayoritariamente las vegetativas relacionadas con el tallo: color, presencia de antocianinas, forma y pubescencia. Adicionalmente, hábito de crecimiento y pubescencia de la hoja tuvieron el mismo valor discriminante que las variables de tallo (Cuadro 6). Este poder discriminante logró separar en el primer eje a las plantas del cultivar Rosita de las plantas de Pepón y Llanero. En el segundo eje, Pepón se diferenció de Rosita y Llanero (Figura 1) por la variable días a la floración, seguida de tres variables relacionadas con el fruto: forma del ápice del fruto, días para la fructificación y ancho del fruto (Cuadro 6). 
Cuadro 5. Promedio y coeficiente de variación de las variables cuantitativas medidas sobre quince plantas de las variedades Rosita, Pepón y Llanero de ají dulce (Capsicum chinense Jacq.) venezolano, en La Vigía, municipio Jiménez, estado Lara, Venezuela. 2017.

Table 5. Mean and variation coefficient of the quantitative variables measured on fifteen plants of the Venezuelan sweet pepper (Capsicum chinense Jacq.) varieties: Rosita, Pepon, and Llanero. La Vigía, Jiménez municipality, Lara state, Venezuela. 2017.

\begin{tabular}{lcccccc}
\hline & \multicolumn{2}{c}{ Rosita } & \multicolumn{2}{c}{ Pepón } & \multicolumn{2}{c}{ Llanero } \\
\cline { 2 - 7 } Variable & Promedio & CV & Promedio & CV & Promedio & CV \\
\hline 2.8. Ancho de la planta (cm) & $32,52 \mathrm{a}$ & 45,73 & $49,60 \mathrm{a}$ & 5,99 & $40,54 \mathrm{a}$ & 40,16 \\
2.9. Longitud del tallo (cm) & $22,41 \mathrm{a}$ & 32,87 & $12,61 \mathrm{ab}$ & 18,22 & $12,30 \mathrm{~b}$ & 27,91 \\
2.10. Diámetro del tallo (cm) & $3,05 \mathrm{a}$ & 21,70 & $3,39 \mathrm{a}$ & 9,39 & $2,99 \mathrm{a}$ & 16,58 \\
2.18. Longitud de la hoja madura (cm) & $8,58 \mathrm{a}$ & 17,83 & $6,90 \mathrm{a}$ & 3,44 & $6,69 \mathrm{a}$ & 13,52 \\
2.19. Ancho de la hoja madura (cm) & $5,30 \mathrm{a}$ & 25,26 & $4,33 \mathrm{ab}$ & 13,32 & $3,56 \mathrm{~b}$ & 14,36 \\
3.1. Días a la floración & $62,50 \mathrm{a}$ & 6,69 & $55 \mathrm{~b}$ & 0 & $61 \mathrm{ab}$ & 6,86 \\
4.1. Días a la fructificación & $76,67 \mathrm{a}$ & 5,33 & $68,33 \mathrm{a}$ & 8,45 & $77 \mathrm{a}$ & 5,81 \\
4.7. Longitud del fruto (cm) & $3,05 \mathrm{~b}$ & 10,80 & $3,52 \mathrm{~b}$ & 14,37 & $6,25 \mathrm{a}$ & 13,03 \\
4.8. Ancho del fruto (cm) & $2,98 \mathrm{ab}$ & 8,62 & $3,40 \mathrm{a}$ & 13,30 & $2,71 \mathrm{~b}$ & 13,95 \\
4.9. Peso del fruto (g) & $6,70 \mathrm{~b}$ & 13,71 & $10,08 \mathrm{a}$ & 4,70 & $11,17 \mathrm{a}$ & 18,19 \\
4.10. Longitud del pedicelo del fruto $(\mathrm{cm})$ & $2,61 \mathrm{~b}$ & 6,02 & $2,69 \mathrm{ab}$ & 3,02 & $3,24 \mathrm{a}$ & 13,57 \\
4.11. Espesor de la pared del fruto $(\mathrm{mm})$ & $1,78 \mathrm{a}$ & 17,37 & $2,22 \mathrm{a}$ & 23,31 & $1,55 \mathrm{a}$ & 22,74 \\
\hline
\end{tabular}

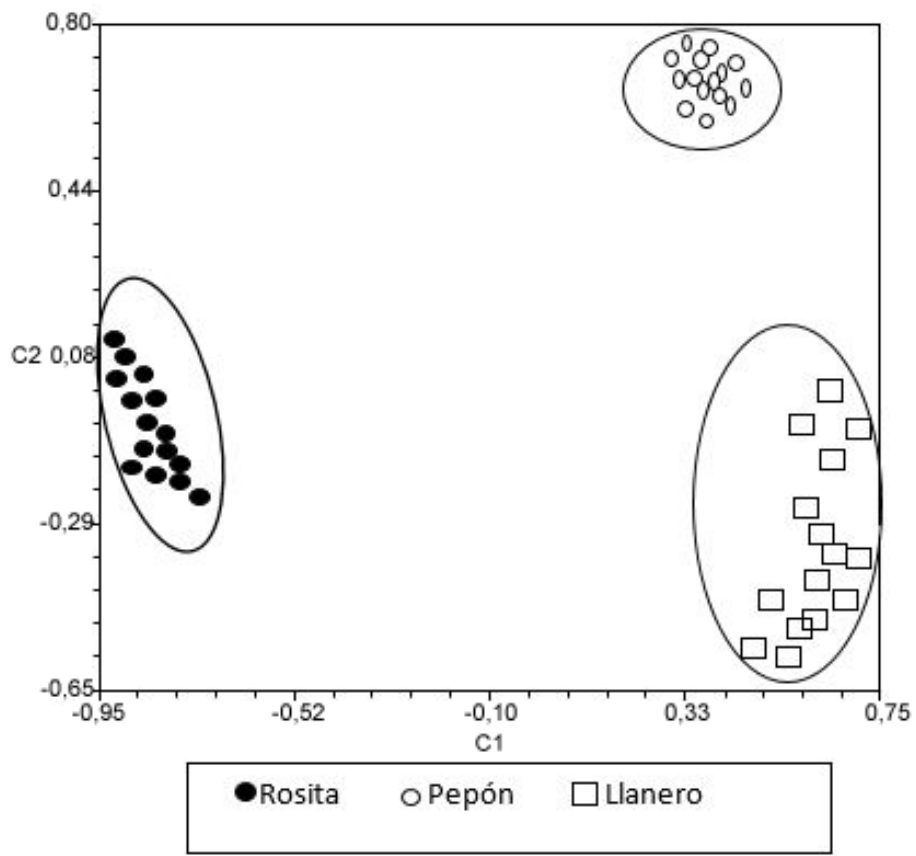

Figura 1. Ordenamiento en un espacio bidimensional de 45 plantas de las variedades Rosita, Pepón y Llanero de aji dulce (Capsicum chinense Jacq.) venezolano, basado en el análisis de componentes principales de 53 atributos morfológicos. La Vigía, municipio Jiménez, estado Lara, Venezuela. 2017.

Figure 1. Arrangement in a two-dimensional space of 45 plants of the Venezuelan sweet pepper (Capsicum chinense Jacq.) varieties: Rosita, Pepón, and Llanero based on principal component analysis of 53 morphological traits. La Vigía, Jiménez municipality, Lara state, Venezuela. 2017. 
Cuadro 6. Autovectores de los dos primeros ejes obtenidos en el análisis de componentes principales de 53 atributos morfológicos en las variedades Rosita, Pepón y Llanero de ají dulce (Capsicum chinense Jacq.) venezolano, en La Vigía, municipio Jiménez, estado Lara, Venezuela. 2017.

Table 6. Eigenvectors of the two first axes from a principal component analysis of 53 morphological traits on the Venezuelan sweet pepper (Capsicum chinense Jacq.) varieties: Rosita, Pepón, and Llanero at La Vigia, Jimenez municipality, Lara state, Venezuela. 2017.

\begin{tabular}{|c|c|c|}
\hline Variables & Eje1 & Eje2 \\
\hline Color del tallo & 0,9706 & 0,1081 \\
\hline Antocianinas en tallo & 0,9706 & 0,1081 \\
\hline Forma del tallo & 0,9706 & 0,1081 \\
\hline Pubescencia del tallo & 0,9706 & 0,1081 \\
\hline Altura & $-0,658$ & 0,5006 \\
\hline Hábito de crecimiento & $-0,9706$ & $-0,1081$ \\
\hline Ancho planta & $-0,0049$ & 0,7199 \\
\hline Longitud del tallo & $-0,8397$ & $-0,0523$ \\
\hline Diámetro del tallo & $-0,2289$ & 0,6463 \\
\hline Densidad de ramificación & 0,9142 & 0,2802 \\
\hline Pubescencia de la hoja & 0,9706 & 0,1081 \\
\hline Longitud de la hoja & $-0,8371$ & 0,1036 \\
\hline Ancho de la hoja & $-0,8393$ & 0,2709 \\
\hline Dias a floración & $-0,2035$ & $-0,8027$ \\
\hline Longitud de las anteras & 0,1772 & $-0,0234$ \\
\hline Longitud del filamento & 0,0217 & $-0,0836$ \\
\hline Días a la fructificación & 0,0272 & $-0,7811$ \\
\hline Rayas antocianínicas & 0,9142 & 0,2802 \\
\hline Color del fruto & 0,8734 & 0,4158 \\
\hline Cuajado del fruto & $-0,4455$ & 0,1033 \\
\hline Color del fruto maduro & 0,9285 & 0,0796 \\
\hline Forma del fruto & $-0,5638$ & 0,6241 \\
\hline Longitud del fruto & 0,7236 & $-0,3265$ \\
\hline Ancho del fruto & $-0,2044$ & 0,7401 \\
\hline Peso del fruto & 0,8081 & 0,2030 \\
\hline Longitud del pedicelo & 0,5425 & $-0,4244$ \\
\hline Espesor de la pared del fruto & 0,0063 & 0,5309 \\
\hline Forma del ápice del fruto en unión con el pedicelo & 0,1996 & 0,6716 \\
\hline $\begin{array}{l}\text { Forma ápice del fruto } \\
\end{array}$ & $-0,3185$ & 0,7832 \\
\hline
\end{tabular}

\section{Discusión}

La variación encontrada en el $42 \%$ de los atributos morfológicos evaluados en tres variedades de $C$. chinense, coincidió con lo indicado por Jarret y Berke (2008), Sudré et al. (2010), Carvalho et al. (2014) y Baba et al. (2016), sobre la amplia variación de esta especie.

$\mathrm{Al}$ considerar los atributos morfológicos evaluadas, de acuerdo con la parte de la planta a la que pertenece según el número de rasgos morfológicos utilizados, la variación en flor y semilla fue la menos adecuada para poder diferenciar a los tres cultivares, ya que de las diecisiete características evaluadas en conjunto en estos órganos, solo 
una presentó variación entre las 45 plantas que conformaron la muestra. De las variables vegetativas evaluadas, las de hoja fueron las menos adecuadas para poder diferenciar a los tres cultivares, ya que tan solo pubescencia en la hoja y ancho de la hoja resultaron con variabilidad, por el contrario, los atributos morfológicos evaluados en tallo variaron en cinco de los seis rasgos morfológicos medidos.

Dos terceras partes de las variables medidas en frutos permitieron la discriminación entre cultivares, entre ellas forma y peso del fruto, tal como también lo reportaron Jarret y Berke (2008), al evaluar exclusivamente caracteres de fruto entre más de 300 introducciones de esta especie, y Baba et al. (2016) al usar catorce descriptores morfológicos en la caracterización y diferenciación de 71 introducciones de $C$. chinense.

En otras especies de Capsicum, tal como en C. frutescens, también se han reportado atributos morfológicos relacionados al tallo y al fruto que han permitido la discriminación entre distintas introducciones, específicamente longitud del tallo, diámetro del tallo, peso del fruto, longitud de fruto y espesor de la pared del fruto (Carvalho et al., 2017; de-la-Cruz-Lázaro et al., 2017).

Al observar en conjunto los veintidós atributos morfológicos que resultaron con variación, se define que la diversidad intravarietal fue pequeña al ser comparada con la diversidad intervarietal; existió una identidad genética definida para cada uno de los tres tipos de ají evaluados ya que no hubo ningún tipo de yuxtaposición de plantas de distintos cultivares en el gráfico bidimensional obtenido por el análisis de componentes principales. Esta situación permitió inferir que las labores de domesticación o las de mejoramiento genético que se hayan dado para cada uno de los cultivares se dieron de manera independiente, o si no fue de esta manera, se mantuvo el aislamiento requerido entre los cultivares, evitando así la mezcla de semillas o la polinización cruzada que eventualmente pudiera ocurrir dentro de $C$. chinense.

Entre las variables categóricas que tuvieron mayor variabilidad estuvieron la forma y el color del fruto, mientras que entre las variables cuantitativas, longitud, ancho y peso del fruto fueron las que presentan mayor variabilidad intercultivar, logrando identificar diferencias estadísticas entre cultivares. Como en ají dulce lo que se comercializa es el fruto, es deseable la situación acá reportada de existir potencial de mejoramiento genético para atributos del fruto, al igual que lo afirmaron Jarret y Berke (2008), basados en la diversidad encontrada para atributos del fruto de $C$. chinense. El ají dulce en Venezuela ha recibido poca atención desde el punto de vista de su mejoramiento genético, y esto se visualizó en que los productores del campo utilizan los mismo tres tipos de ají desde hace varias décadas.

Los resultados reportados en el presente trabajo indicaron el potencial de abordar actividades de mejoramiento genético dentro de la especie $C$. chinense, y así superar una de las desventajas que Moses y Umaharan (2012) indicaron para la producción del cultivo: los productores del campo usan tipos nativos que no han sido debidamente caracterizados, por lo cual no se abordan actividades para la obtención de nuevos cultivares.

\section{Conclusiones}

De las 53 características morfológicas evaluadas, veintidós permitieron identificar inequívocamente a cada uno de los tres cultivares de ají dulce evaluados. La diferencia entre cultivares fue determinada con análisis de componentes principales, sin ningún tipo de sobreposición entre plantas de diferentes cultivares, permitió concluir que la variación intercultivar fue mucho mayor que la variación intracultivar.

\section{Literatura citada}

Andrade, S., y H. Laurentin. 2015. Efecto del nitrato de potasio sobre la germinación de semillas de tres cultivares de ají dulce (Capsicum chinense Jacq.). Rev. Unell. Cienc. Tec. 33:25-29. 
Baba, V., K. Rocha, G. Gomes, C. Ruas, P. Ruas, R. Rodrigues, and L. Azeredo. 2016. Genetic diversity of Capsicum chinense accessions based on fruit morphological characterization and AFLP markers. Genet. Res. Crop Evol. 63:1371-1381. doi:10.1007/s10722-015-0325-4

Cardoso, R., C. Ruas, R. Giacomin, P. Ruas, E. Ruas, R. Barbieri, R. Rodrigues, and L. Goncalves. 2018. Genetic variability in Brazilian Capsicum baccatum germplasm collection assessed by morphological fruit traits and AFLP markers. PLoS ONE 13(5):e0196468. doi:10.1371/journal.pone.0196468

Carvalho, S., L. Bianchetti, C. Ragassi, C. Ribeiro, F. Reifschneider, G. Buso, and F. Faleiro. 2017. Genetic variability of a Brazilian Capsicum frutescens germplasm collection using morphological characteristics and SSR markers. Genetics Mol. Res. 16(3):gmr16039689. doi:10.4238/gmr16039689

Carvalho, S., C. Ragassi, L. Bianchetti, F. Reifschneider, G. Buso, and F. Faleiro. 2014. Morphological and genetic relationships between wild and domesticated forms of peppers (Capsicum frutescens L. and C. chinense Jacquin). Genetics Mol. Res. 13:7447-7464. doi:10.4238/2014.September.12.11

Cedeño, L., C. Carrero, y R. Jaimez. 2003. Pudrición basal del ají dulce por Haematonectria haematococca en el estado Mérida, Venezuela. Interciencia 28:590-592.

Colivet, J., G. Belloso, y E. Hurtado. 2006. Comparación del efecto inhibidor de extractos de ají dulce (Capsicum chinense) sobre el crecimiento de Escherichia coli y Bacillus sp. Saber 18:168-173. http://ojs.udo.edu.ve/index.php/saber/article/ view/335/229.

de-la-Cruz-Lázaro, E., C. Marquez-Quiroz, R. Osorio-Osorio, P. Preciado-Rangel, y C. Márquez-Hernández. 2017. Caracterización morfológica in situ de chile silvestre Pico de paloma (Capsicum frutescens) en Tabasco, México. Acta Universitaria 27:10-15. doi:10.1517/au.2017.1083

Eldeen, I., M. Effendy, and T. Tengku-Muhammad. 2016. Ethnobotany: challenges and future perspectives. Res. J. Med. Plants 10:382-387. doi:10.3923/rjmp.2016

Eshbaugh, W.H. 1980. The taxonomy of the genus Capsicum (Solanaceae). Phytologia 47:153-166.

Gil-Marín, J., N. Montaño-Mata, y R. Plaza. 2012. Efecto del riego y la cobertura del suelo sobre la productividad de dos cultivares de ají dulce. Bioagro 24:143-148.

Govindaraj, M., M. Vetriventhan, and M. Srinivasan. 2015. Importance of genetic diversity assessment in crop plants and its recent advances: an overview of its analytical perspectives. Genet. Res. Int. 2015:431487. doi:10.1155/2015/431487

Hallewood, M., T. Chiurugwi, R. Hamilton, B. Kurtz, E. Marden, E. Welch, F. Michiels, J. Mozafari, M. Sabran, N. Patron, P. Kersey, R. Bastow, S. Dorius, S. Dias, S. McCouch, and W. Powell. 2018. Plant genetic resources for food and agriculture: opportunities and challenges emerging from the science and information technology revolution. New Phytol. 217:14071419. doi:10.1111/nph.14993

Holmes, M., T. Hammond, G. Wogan, R. Walsh, K. Labarbera, E. Wommack, F. Martins, J. Crawford, K. Mack, L. Bloch, and M. Nachman. 2016. Natural history collections as windows on evolutionary processes. Mol. Ecol. 25:864-881. doi: $10.1111 /$ mec.13529

IPGRI (International Plant Genetic Resources Institute), AVRDC (Asian Vegetable Research and Development Center), and CATIE (Centro Agronómico Tropical de Investigación y Enseñanza). 1995. Descriptors for Capsicum (Capsicum spp.). IPGRI, Rome, Italy, AVRDC, Taipei, Taiwan, and CATIE, Turrialba, CRI.

Jaimez, R. 2000. Crecimiento y distribución de la materia seca en ají dulce bajo condiciones de déficit de agua. Agron. Trop. 50:189-200. 
Jaimez, R., N. Nava, Y. Rivero, y K. Trompiz. 2002. Efecto de diferentes intensidades de poda sobre la dinámica de floración y producción de ají dulce (Capsicum chinense Jacq.). Rev. Fac. Agron. (LUZ) 19:132-139.

Jarret, R., and T. Berke. 2008. Variation for fruit morphological characteristics in a Capsicum chinense Jacq. Germplasm collection. HortScience 43:1694-1697. doi:10.21273/HORTSCI.43.6.1694.

Laurentin, H. 2009. Data analysis for molecular characterization of plant genetic resources. Genetic Res. Crop Evol. 56:277292. doi:10.1007/s10722-008-9397-8

Moses, M., and P. Umaharan. 2012. Genetic structure and phylogenetic relationships of Capsicum chinense. J. Am. Soc. Hortic. Sci. 137:250-262. doi:10.21273/JASHS.137.4.250

Montaño, N. 2000a. Efecto de la edad de trasplante sobre el rendimiento de tres selecciones de ají dulce (Capsicum chinense Jacq.). Bioagro 12:55-59.

Montaño, N. 2000b. Evaluación de tres métodos de producción de plántulas de ají dulce (Capsicum chinense Jacq.) en Jusepín, estado Monagas. Bioagro 12:81-84.

Olatunji, T., and A. Afolayan. 2019. Contribution to the classification of Capsicum annuum L. and Capsicum frutescens L. in West Africa using morphological traits. Notulae Botanicae Horti Agrobotanici Cluj-Napoca 47:135-142. doi:10.15835/ nbha47111204

Pastrán, F. 2019. Desarrollo cultural canario-venezolano desde la expresión humana, religiosa, deportiva, gastronómica y agrícola. Rev. Chakiñan Cienc. Soci. Humanidades 7:118-134.

Shirasawa, K., T. Ban, N. Nagata, and T. Murakana. 2019. Impact of genomics on Capsicum breeding. In: N. Ramchiary, and C. Kole, editors, The Capsicum genome. Compendium of Plant Genomes. Springer, Berlin, DEU. p. $209-219$. doi:10.1007/978-3-319-97217-6_13

Sudré, C., L. Goncalves, R. Rodrigues, A. do-Amaral-Júnior, E. Riva-Souza, and C. Bento. 2010. Genetic variability in domesticated Capsicum spp as assessed by morphological and agronomic data in mixed statistical analysis. Genetics Mol. Res. 9:283-294. doi:10.4238/vo19-1gmr698

Upadhyaya, H.D., C.L. Gowda, and D.V. Sastry. 2008. Plant genetic resources management: collection, characterization, conservation and utilization. J. SAT Agric. Res. 6:1-16. 